\title{
Estudos sistêmico-funcionais da tradução
}

Silvana Maria de Jesus*

Resumo: Este artigo introduz a interface entre os estudos da tradução e a linguística sistêmicofuncional, desenvolvida por Michael A. K. Halliday, como uma abordagem produtiva para se investigar o fenômeno tradutório. Esta interface pode ser considerada em duas fases, tendo como marco divisório a publicação da obra editada por Steiner e Yallop (2001) e a proposta dos estudos multilíngues apresentada por Matthiessen (2005). Tem como objetivo, ainda, apresentar os pressupostos teóricos básicos da linguística sistêmico-funcional, relacionando sua aplicabilidade aos estudos da tradução dentro de uma perspectiva teórica-prática. Os trabalhos desenvolvidos sob a ótica desta interface corroboram a possibilidade de se pensar a tradução a partir de uma teoria linguística que inclua em seu escopo o uso da linguagem sob uma perspectiva social e semiótica.

Palavras-chave: Estudos da tradução; Linguística sistêmico-funcional; análise contrastiva.

\begin{abstract}
This paper introduces the interface between translation studies and the systemic functional linguistics developed by Michael A. K. Halliday as a productive approach to investigate the phenomenon of translation. This interface can be considered in two phases, with the landmark publication of the book edited by Steiner and Yallop (2001) and the proposal of multilingual studies by Matthiessen (2005). It also aims at presenting the basic theoretical principles of systemic functional linguistics and its applicability to translation studies within a theoretical and practical perspective. The works developed under the perspective of this interface corroborate the possibility of thinking about translation using a linguistic theory which includes in its assumptions the use of language in a social and semiotic perspective.
\end{abstract}

Keywords: translation studies; Systemic Functional Linguistics; contrastive analysis.

\section{A interface entre a linguística sistêmico-funcional e os estudos da tradução}

A interface entre os estudos da tradução e a linguística sistêmico-funcional (doravante $\mathrm{LSF}^{1}$ ) tem sido mapeada no Brasil por diversos autores - Vasconcellos (1997), Pagano e Vasconcellos (2005), Jesus e Pagano (2007), Figueredo (2007).

\footnotetext{
* Doutorado em Linguística Aplicada - Tradução, UFMG, 2008. Pós-doutorado em Tradução/Linguística de corpus, Alemanha, UdS, 2009. Professora Adjunto do Curso de Tradução, ILEEL, UFU.

${ }^{1}$ A linguística sistêmico-funcional (LSF) é uma teoria que explica a linguagem enquanto fenômeno semiótico e social, sendo Halliday seu principal teórico. A gramática sistêmico-funcional (GSF) é a obra base que reúne os pressupostos desta teoria. Embora se utilize o termo GSF ela ainda não foi traduzida para o português, sendo que a obra em inglês denomina-se An introduction to functional grammar (Halliday e Matthiessen, 2004, $3^{\text {a }}$ edição). Outros aspectos teóricos da linguística sistêmica podem ser encontrados em outras obras dos autores e de outros sistemicistas, como Halliday (1964), Eggins (1994), Thompson (1996), Martin (1997), Halliday e Matthiessen (1999). Neste artigo, usa-se LSF para se referir à teoria em geral e GSF para se referir à gramática.
} 
Desde o início da formulação da teoria sistêmica, Halliday dedicou atenção ao fenômeno tradutório, visto como um dos fenômenos possíveis dentro do campo da linguagem. Catford foi um dos pioneiros em desenvolver uma teoria de tradução com base na LSF nos anos 1960, interesse que foi retomado posteriormente por outros teóricos da tradução (cf. VASCONCELLOS, 1997 e PAGANO; VASCONCELLOS, 2005).

Pode-se considerar que a publicação da obra Exploring translation and multilingual text production: beyond content (STEINER; YALLOP, 2001), marca o início de uma nova fase na interface entre a LSF e a tradução. Nesta obra, diversos autores, entre eles o próprio Halliday, observam o fenômeno tradutório sob a perspectiva da LSF. Esta nova fase, portanto, é marcada pelo interesse dos sistemicistas pela tradução, fenômeno que estes consideram diretamente relacionado à produção textual multilíngue.

O termo produção textual multilíngue merece algumas considerações. Matthiessen (2005) defende que as diferentes atividades que existem dentro e ao redor da linguística, tais como o ensino e a aprendizagem de línguas, a formação de tradutores, os estudos da tradução e outras, poderiam ser agrupadas dentro de um espaço teórico que poderíamos chamar de estudos multilíngues (multilingual studies), visto que estas atividades comprovam a diversidade linguística que existe no mundo e poderiam beneficiar-se mutuamente se houvesse um maior intercâmbio entre estas áreas, que geralmente se desenvolvem de forma isolada. Matthiessen (2007) pondera que o linguista inglês J. R. Firth criou as bases para uma teoria linguística que abre espaço para a descrição das especificidades de cada língua e seus sistemas em oposição à perspectiva simplista de universais em que se baseiam as gramáticas tradicionais. Firth, com suas pesquisas de bases antropológicas, destaca a função comunicativa da linguagem e a importância de se estudar a língua a partir de seu contexto de uso, por ele chamado de contexto de situação. Firth elaborou uma teoria contextual do significado, que ele definia como "a função de uma forma linguística em um contexto"2 (JOSEPH; LOVE; TAYLOR, 2001:62). Os trabalhos de Halliday, Hoey e Sinclair, entre outros, contribuem para a expansão da linha neo-firthiana.

\footnotetext{
2 "Meaning is a function of a linguistic form in a context" (JOSEPH; LOVE; TAYLOR, 2001:62, tradução nossa).
} 
Sob esta perspectiva funcional e multilíngue, os sistemicistas se ocupam de repensar os conceitos de tradução e de equivalência, buscando descrever e comparar textos e sistemas. Outras características importantes desta nova fase são o amadurecimento do processo de análise textual a partir da LSF e o uso de recursos computacionais que facilitam a análise de corpus em termos probabilísticos. Destaca-se ainda, a perspectiva contrastiva, não apenas entre línguas, mas também entre as características de textos traduzidos e textos não-traduzidos, como por exemplo, o trabalho de Jesus (2008), que utiliza a LSF para analisar um corpus combinado ${ }^{3}$. Jesus (2008) descreve os significados realizados por SAY ${ }^{4}$ em inglês e DIZER em português, contrastando o uso destes itens lexicais nas duas línguas; constatou-se uma maior ocorrência de DIZER em expressões modalizadoras e de SAY em discurso direto. Jesus (2008) contrasta também o uso de DIZER em textos originalmente produzidos em português e textos traduzidos para o português, observando a influência do uso de SAY nos textos originais, neste caso, em inglês, sobre o uso de DIZER em textos traduzidos, projetando uma frequência maior de DIZER em discurso direto nas traduções. A mesma análise foi feita em relação à SAY.

Portanto, cabe aqui retomar as bases da construção da interface entre a LSF e a tradução, focalizando a nova fase e apresentando os pressupostos básicos da LSF como fundamentação para a análise textual.

Halliday et al. $\left(1974\right.$, p. 137) ${ }^{5}$ consideram que uma teoria de tradução se insere entre as diversas teorias no campo da linguística comparada, mais especificamente, a linguística descritiva comparada, que desenvolve teorias e métodos para a comparação entre as línguas. Essa descrição diz respeito ao modo como duas línguas são relacionadas ou a "comparar as línguas de acordo com o modo como funcionam".

Os autores (HALLIDAY et al., 1974, p. 138) apontam dois princípios fundamentais para a linguística descritiva comparada: i) descrever antes de comparar, e ii) comparar padrões e não línguas na sua totalidade. Mencionam ainda três etapas para

\footnotetext{
${ }^{3}$ Um corpus combinado, ou corpus bi-direcional, é um corpus formado de originais e traduções nas duas direções. Neste caso, Jesus (2008) utiliza um corpus com textos originais em inglês e suas traduções para o português e textos originais em português e suas traduções para o inglês.

4 Conforme os pressupostos da linguística de corpus, usam-se maiúsculas para indicar que todas as formas do vocábulo foram consideradas. Nesta pesquisa, foram analisadas todas as formas, incluindo os casos em que os itens lexicais não funcionavam como verbos.

${ }^{5}$ O original é de 1964, utiliza-se neste artigo a tradução de 1974, feita por Myriam Freire Morau.
} 
uma descrição: i) descrever um padrão em cada língua, separadamente, ii) estabelecer se estes padrões são comparáveis, e iii) compará-los. Entende-se por padrão todas as palavras e estruturas regularmente associadas a uma palavra e que contribuem para a sua significação (FRANCIS; HUNSTON, 2000:37).

Segundo Halliday et al. (1974, p. 140), a tradução contribui para a segunda etapa do processo, a de estabelecer padrões de comparação, pois "se os elementos não são, ao menos algumas vezes, equivalentes na tradução, não vale a pena compará-los”. É necessário, segundo os autores, observar as probabilidades de equivalência de tradução. Os autores consideram que "a comparação é a apresentação das diferenças com relação a um pano de fundo formado pelas semelhanças" e que "uma semelhança aparente esconde importantes diferenças" (p. 151). Neste contexto, introduzem o conceito de equivalências, no plural, considerando que as palavras e as categorias têm "uma série de equivalentes potenciais em uma escala de probabilidades" (p. 151). Os autores não aprofundam a discussão sobre o conceito de equivalência, mas Halliday volta a focar a questão dos vários equivalentes potencias em uma publicação posterior (Halliday, 1992), apontando para a noção de equivalência lexical. Nos termos de Halliday (1992:16), "um item $\mathrm{X}$ na língua do original tem um grupo de itens

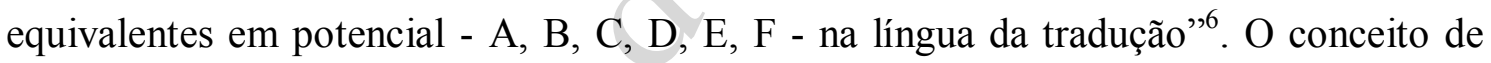
probabilidades é retomado por Catford (1980) ${ }^{7}$ e desenvolvido por Matthiessen (2001).

Catford (1980, p. 1) explicita que sua teoria linguística da tradução baseia-se na teoria de Halliday, mas esclarece que simplifica esta teoria, inclusive não dando o devido aprofundamento a algumas categorias, como por exemplo, o tratamento de níveis linguísticos. Ressalta-se que o trabalho de Catford foi pioneiro ao iniciar esta interface entre os estudos da tradução e a LSF, levando-o a formular os conceitos de probabilidades condicionadas e incondicionadas de tradução.

Como apontam Pagano e Vasconcellos (2005, p. 185):

Embora o modelo proposto por Catford sofra das limitações apontadas (...) e não tenha se mantido como arcabouço teórico e metodológico

\footnotetext{
6 "You are aware that an item $\mathrm{X}$ in the source language has a potential equivalence of items A, B, C, D, E, F in the target language" (Halliday, 1992:16, tradução nossa)

${ }^{7}$ O original é de 1965 , utiliza-se nesta tese a tradução de 1980 , feita pelo Centro de especialização de tradutores de inglês da PUC-Campinas.
} 
frutífero nos estudos da tradução, seu detalhamento se justifica por representar o pioneirismo nesta interface e pelo fato de as categorias propostas terem se mantido como referência (mesmo negativa) na área. Cumpre agora, explorar a interface em estudos mais recentes, realizados a partir do final da década de 1960, que marca a orientação funcional da linguística sistêmica.

Para uma visão geral de trabalhos que se basearam nesta interface, remeto o leitor a Vasconcellos (1997) e Pagano e Vasconcellos (2005), que mapeiam os principais trabalhos publicados nos anos 1980 e 1990. Importa mapear aqui alguns trabalhos da nova fase, focalizando a perspectiva da LSF sobre a tradução. Destacam-se os trabalhos publicados na obra Exploring translation and multilingual text production: beyond content (STEINER; YALLOP, 2001), apontados a seguir.

Halliday (2001, p. 13) considera que quando se pensa em uma teoria geral de tradução pode-se focar em dois aspectos. A preocupação do linguísta seria uma teoria sobre a natureza do processo de tradução e as questões envolvendo as ligações entre textos em relação tradutória. Por outro lado, um tradutor estaria interessado em uma teoria que explicasse (e não prescrevesse) como uma tradução deveria ser, como avaliar a qualidade de uma tradução e como produzir uma tradução eficaz. Obviamente, Halliday (2001, p. 14) reconhece que a avaliação de uma tradução depende de uma variedade de fatores, bastante complexos, e que o valor de uma tradução é um valor relativo, dependendo do nível (lexicogramatical, semântico ou contextual) em que se espera maior equivalência.

Gregory (2001) utiliza uma perspectiva sócio-cognitiva e enfatiza a necessidade de uma teoria que integre forma e significado, estrutura e uso, a partir da perspectiva da LSF de que os recursos formais da língua servem à representação da experiência humana.

Matthiessen (2001), pesquisador dedicado ao desenvolvimento da sistêmica, retoma, nesta obra, os conceitos apresentados por Halliday et al (1974) e Catford (1980), dando ênfase à análise multi-contextualizada da tradução e das probabilidades condicionadas das várias equivalências. $\mathrm{O}$ autor considera as probabilidades dentro do que ele denomina de ambientes contextuais da tradução (environments of translation), a partir de sua perspectiva de tradução enquanto (re)construção de significados. Enquanto linguista e não sendo teórico da tradução, Matthiessen (p. 41) situa seu interesse no 
tema em uma perspectiva de "questões relacionadas à comunicação multilíngue" e analisadas a partir dos pressupostos da LSF. O autor define a tradução como "um processo de construção da experiência enquanto significado (...) uma experiência construída enquanto significado em um sistema linguístico é (re)construída linguisticamente em outro" ${ }^{\prime 8}$ (p. 51).

House (2001) apresenta um modelo de avaliação da qualidade de uma tradução, a partir da análise das relações entre o texto original e o texto traduzido, procurando estabelecer critérios para que se possa afirmar se um texto é ou não uma tradução ou um outro tipo de produção textual. Em sua discussão sobre o conceito de equivalência, a autora (p. 134) aponta que a tradução é uma produção textual que apresenta um vínculo duplo. Por um lado, tem relações com um texto original em outra língua e, por outro, com o potencial comunicativo da comunidade receptora. Sob esta perspectiva, House apresenta dois tipos de tradução: a encoberta (covert), que se apresenta na comunidade receptora com o status de um original, e a manifesta (overt), que, devido às suas fortes ligações com o texto e a cultura do original não se dirige especificamente ao leitor da comunidade receptora. A autora explora estes conceitos em uma discussão sobre a qualidade de tradução.

Steiner (2001) discute o conceito de tradução, relacionando-o ao de registro e explorando os tipos de relações entre textos, propondo que o texto traduzido apresenta diferenças sistemáticas em relação a outros tipos de textos, caracterizando-se como um registro per se.

Yallop (2001), por sua vez, discute o conceito de tradução a partir de uma perspectiva filosófica de que não existe identidade absoluta em situação alguma. $O$ autor defende a dificuldade de se traçar limites entre o que se define como tradução e adaptação, considerando-se que a equivalência não é uma relação fixa, mas que se define a partir de vários aspectos que permitem determinar um tipo de semelhança que seja admitido em determinada situação.

Teich (2001) apresenta um modelo de análise contrastiva dos recursos linguísticos envolvidos na tradução. A LSF é usada como recurso para se contrastar textos, observando-se semelhanças e diferenças que ampliam os conceitos de tradução e

${ }^{8 ،}$ “... a process of construing experience as meaning (...) (experience construed as) meaning in one system is (re)construed as meaning in another". (Mhatthiessen, 2001:51, tradução nossa, grifos do autor). 
de equivalência e apontam um caminho para a investigação das propriedades dos textos traduzidos.

O trabalho desenvolvido por Teich $(2001,2003)$ é de particular interesse na interface entre os estudos da tradução e a LSF, pois a autora propõe um modelo de análise contrastiva que inclui a investigação das propriedades dos textos traduzidos. A autora aplica a LSF em métodos específicos de análise.

O modelo apresentado por Teich (2003, p. 29) tem como pressupostos: i) o uso de uma teoria linguística abrangente que permita interpretar os dados encontrados e comparar textos e sistemas linguísticos, observando suas semelhanças e diferenças; ii) a análise contrastiva baseada em descrições linguísticas existentes para fornecer informações sobre as semelhanças e diferenças entre os sistemas linguísticos em análise; iii) a escolha de características linguísticas que sejam significativas para as hipóteses que estão sendo testadas; e iv) o desenvolvimento de uma análise quantitativa baseada em corpora.

A teoria linguística considerada apropriada para o modelo é a LSF devido à sua perspectiva funcional e multi-dimensional que permite análises linguísticas abrangentes integrando forma e significado. Uma síntese dos pressupostos teóricos desta teoria é apresentada na próxima seção.

Quanto aos métodos, Teich propõe que a análise contrastiva seja feita a partir de descrições linguísticas existentes para as línguas a serem contrastadas. Por exemplo, a autora (TEICH, 2003, p. 66) utiliza, para os aspectos que ela deseja contrastar no par linguístico inglês-alemão, descrições sistêmicas existentes e descrições feitas a partir de outras teorias, mas que encontram ressonância nas categorias da LSF. Neste sentido, a autora aponta que é necessário escolher características linguísticas que sejam relevantes para as hipóteses sob investigação e que a análise deve utilizar textos traduzidos e não-traduzidos nas duas direções do par linguístico envolvido, sendo que os resultados devem ser apresentados quantitativa e qualitativamente.

Com este modelo, Teich $(2003$, p. 1) propõe investigar as seguintes questões, em relação ao par linguístico inglês-alemão: 
1- Qual é a relação entre textos não-traduzidos em um mesmo registro ${ }^{9}$ em duas línguas distintas?

2- Qual é a relação entre um texto original na língua A e a tradução deste texto na língua $\mathrm{B}$ ?

3- Qual é a relação entre textos não-traduzidos em uma dada língua e textos traduzidos nesta mesma língua? Eles apresentam os mesmos padrões em relação a uma dada característica linguística ou seus padrões são diferentes?

Este modelo mostra-se bastante produtivo, especialmente do ponto de vista da interface entre a LSF e os estudos da tradução, pois permite descrever e contrastar padrões linguísticos tanto entre os originais e as traduções (análise bilíngue) como entre os textos traduzidos e os não-traduzidos em uma mesma língua (análise monolíngue). Para este tipo de análise, faz-se necessário o uso de um corpus combinado, que apresente textos traduzidos e não-traduzidos nas duas línguas em análise (cf. JESUS, 2008).

A LSF é a teoria utilizada para a análise textual, primeiro passo para a descrição linguística, que, por sua vez, permite a comparação entre textos e sistemas. Apresentamse, portanto, os principais fundamentos desta teoria.

\section{Principais pressupostos teóricos da linguística sistêmico-funcional}

Halliday e Matthiessen (2004, p. 64) concebem a construção de significados como uma operação que pode ser analisada de acordo com "três linhas" ou três metafunções: experiencial (foco na representação), interpessoal (foco na interação) e textual (foco na organização textual). Cada uma destas metafunções possui padrões léxico-gramaticais próprios que realizam os diferentes significados. A GSF (HALLIDAY; MATTHIESSEN, 2004, p. 61) considera ainda uma quarta metafunção,

${ }^{9} \mathrm{O}$ conceito de registro é complexo e varia entre diferentes autores. Teich utiliza os conceitos de Biber, Halliday e Matthiessen (apud TEICH 2003, p. 23, tradução nossa): "registros são categorias de textos facilmente reconhecíveis pelos falantes de uma língua, ou, em termos técnicos, são grupos de textos realizados por uma ocorrência relativamente alta ou baixa de determinadas características léxicogramaticais". 
que não é visível na oração e sim no complexo oracional. É a metafunção lógica, referente à construção lógica ou relacional entre as orações.

O conceito de função e de metafunção para a LSF merece ser destacado. Como explicam Halliday e Hasan (1993, p. 23):

Cada sentença em um texto é multifuncional; mas, não no sentido de que se pode apontar para um elemento e dizer que tal elemento tem uma função específica. Os significados estão imbricados, formando uma rede, de forma que, para compreendê-los, não olhamos cada um isoladamente; ao contrário, olhamos para a rede como um todo, sob diferentes ângulos, cada ângulo contribuindo para a interpretação global. Este é o princípio fundamental de uma abordagem funcional. ${ }^{10}$

O termo metafunção é utilizado para se explicitar esta perspectiva multifuncional, visto que a palavra função, em outras perspectivas teóricas, é utilizada para expressar simplesmente finalidade ou modo de uso da linguagem. Na perspectiva da LSF, este aspecto funcional é intrínseco à linguagem, visto que o modo como a linguagem se constitui está diretamente vinculado às funções a que ela serve (HALLIDAY; MATTHIESSEN, 2004, p. 31).

A análise da metafunção experiencial ocorre por meio do sistema de transitividade, que possibilita a representação da realidade através de Processos, Participantes e Circunstâncias ${ }^{11}$. Existem, na LSF, três Processos principais: mental, relacional e material. Processos materiais representam o mundo externo ao falante; Processos mentais representam o mundo interno e os Processos relacionais, estabelecem uma relação identitária ou atributiva entre dois seres ou entidades. No limite entre Processos materiais e mentais estão os Processos comportamentais, que representam as ações do corpo e aquelas motivadas pela consciência ou estados fisiológicos. Entre Processos mentais e relacionais encontram-se os Processos verbais, que representam as

\footnotetext{
${ }^{10}$ "Every sentence in a text is multifunctional; but not in such a way that you can point to one particular constituent or segment and say this segment has just this function. The meanings are woven together in a very dense fabric in such a way that, to understand them, we do not look separately at its different parts; rather, we look at the whole thing simultaneously from a number of different angles, each perspective contributing towards the total interpretation. That is the essential nature of a functional approach." (HALLIDAY; HASAN, 1993, p. 23, tradução nossa).

${ }^{11}$ A GSF segue a convenção de se grafar com a primeira letra em maiúscula os nomes de funções e toda a palavra em maiúsculas para os nomes de sistemas (HALLIDAY; MATTHIESSEN, 2004, p. 113, notas).
} 
relações discursivas. E entre os relacionais e materiais, estão os Processos existenciais, que representam fenômenos que existem ou acontecem.

A metafunção interpessoal representa os recursos da língua para o estabelecimento de relações sociais, realizada pelo sistema de MODO. A terceira metafunção - a textual - diz respeito à criação do discurso no aspecto organizacional da mensagem e é realizado na organização temática da informação.

Embora separadas didaticamente, as três metafunções não representam aspectos isolados da construção de significado, constituindo-se mais propriamente em diferentes perspectivas, ou, como colocam Halliday e Matthiessen (2004, p. 64), são "três linhas de significado, cada uma expressando um tipo de organização semântica diferente”.

Neste contexto, "a gramática é vista como uma rede de escolhas significativas e interrelacionadas" (HALLIDAY; MATTHIESSEN, 2004, p. 31). A LSF analisa este imbricamento de significados através de diversos parâmetros: ordem (rank), estratificação (stratification), metafunção (metafunction), realização (realization) e instanciação (instantiation).

A ordem diz respeito à hierarquia entre as unidades formais. A língua se organiza hierarquicamente em uma escala crescente: morfemas, palavras, grupos, orações e orações complexas. A estratificação é a organização da linguagem em estratos: fonético, fonológico, léxico-gramatical, semântico e contextual. A realização refere-se às relações entre os estratos; considera-se que o significado possui dois aspectos: a expressão (estrato fonológico) e o conteúdo (estratos semântico e léxicogramatical). As metafunções organizam o conteúdo em seu aspecto funcional, que, como foi dito, relacionam-se à construção da experiência humana (metafunção experiencial), ao desempenho de relações sociais (metafunção interpessoal) e à construção do discurso (metafunção textual). Finalmente, a instanciação considera a relação entre o sistema linguístico e o texto (ou instância), seja ele oral ou escrito (cf. HALLIDAY, 2001, p. 15).

A unidade de análise das metafunções é a oração, visto que ela é "o canal principal de energia gramatical" (HALLIDAY; MATTHIESSEN, 2004, p. 31). A LSF também considera aspectos internos e externos da oração (below and above the clause), como os grupos e as relações entre as orações, respectivamente. Considera ainda 
questões de coesão e de retórica, bem como aspectos que extrapolam os limites da oração, como, por exemplo, as metáforas gramaticais.

A LSF adota, portanto, uma perspectiva trinocular (HALLIDAY; MATTHIESSEN, 2004, p. 31), observando uma unidade léxico-gramatical no nível em que ela se encontra, ou seja, em uma perspectiva “do mesmo nível” (from around), mas também em uma perspectiva "de cima" (from above), ou seja, do nível semântico, e "de baixo" (from below), do nível lexical ou fonológico.

A LSF adota também uma perspectiva probabilística, a partir da visão de que a linguagem segue padrões reconhecíveis. Como apontam Halliday et al. (1974, p. 165):

(...) os fatos linguísticos não são casuais, mas obedecem a padrões reconhecíveis. Pode-se julgar os padrões como predições sobre os fatos linguísticos, predições que distinguem primeiramente entre o que é possível e o que é impossível, e em seguida, dentro do que é possível, mostram o que é mais provável e o que é menos provável.

O conceito de padrões textuais tem importantes implicações para as análises linguísticas e também para a tradução. A LSF propõe uma teoria para definir o que sejam padrões textuais e explicar a variação destes a partir de uma visão da gramática como um sistema probabilístico. Por gramática probabilística Halliday (1991, p. 31) entende que a gramática de uma língua é composta por sistemas fechados com opções limitadas, sendo que estas opções podem ocorrer de forma equivalente, ou seja, em proporções iguais, ou uma opção pode ser preponderante sobre a outra (equiprobable 0.5:0.5 ou skew 0.9:0.1); daí os conceitos de marcado e não-marcado.

O que esta visão probabilística da gramática propõe é que os padrões podem ser explicados em termos de uma análise qualitativa das opções do sistema - a escolha entre as características A ou B (either this or that) - associada a uma análise quantitativa destas opções - A ocorre mais / B ocorre menos (more like this / less like that), como apontam Nesbitt e Plum (1988, p. 8).

Um exemplo de aplicação do modelo probabilístico é o trabalho de Nesbitt e Plum (1988), em que os autores analisam a existência de padrões nas relações entre orações complexas em diferentes tipos textuais, correlacionando o sistema de taxe (parataxe e hipotaxe) com o das relações lógico-semânticas (projeção e expansão).

Os autores mostram, por exemplo, que o uso de parataxe está estatisticamente correlacionado à projeção de uma locução (discurso direto ou citação), em contraste 
com o uso de hipotaxe que está estatisticamente correlacionado à projeção de ideia (discurso indireto ou relato); também mostram como a projeção de uma locução através de parataxe ou hipotaxe varia segundo o tipo textual seja uma narrativa, uma anedota ou um comentário. Em outros termos, dada a escolha sistêmica qualitativa entre parataxe ou hipotaxe, tem-se, consequentemente, determinadas opções quantitativas, que em termos mais simples seriam: i) parataxe: a projeção de uma locução ocorre mais, a projeção de uma idéia ocorre menos; ii) hipotaxe: a projeção de uma ideia ocorre mais, a projeção de uma locução ocorre menos.

Outro conceito importante na LSF e relevante para a tradução é o de agnação, pois além de estabelecer o que é possível e o que é mais ou menos provável na língua, também é necessário descrever as relações existentes entre as várias possibilidades. A palavra agnação (agnation) e seus derivados agnado ou agnato vêm do latim agnati e significa parentesco. O termo foi usado por Gleason $(1965$, p. 202) para definir as relações entre sentenças que possuem um vocabulário básico em comum, mas estruturas gramaticais distintas.

O conceito de agnação é importante na teoria sistêmica e postula que a gramática é um sistema, uma rede de escolhas interligadas que devem ser vistas "de cima" e explicadas em função de suas inter-relações e não apenas de suas estruturas. Inclui ainda a noção de que as diferentes formas de se expressar uma mesma unidade semântica não são meras variáveis, como se fossem apenas maneiras diferentes de se dizer a mesma coisa. Pelo contrário, as diferentes opções acarretam variações semânticas, tornando-as assim, formas agnatas (HALLIDAY; MATTHIESSEN, 2004, p. 31).

Matthiessen (2001, p. 83) aponta que o conceito de agnação é importante para a tradução porque dado um item $\mathrm{X}$ na língua do original, este item possui vários agnatos dentro do potencial do sistema da língua do original. O tradutor pode optar por traduzir o item X ou um dos seus agnatos. Qualquer que seja a opção do tradutor, o item correspondente também possui a sua rede de agnatos no potencial do sistema da língua da tradução, relacionados ao item X e seus agnatos.

Considerando-se o eixo paradigmático e o eixo sintagmático em torno dos quais a língua se organiza, nota-se que as opções no eixo sintagmático estão em relação estrutural de contraste entre os elementos, ao passo que as opções no eixo paradigmático baseiam-se em relações associativas de similitude. Por exemplo, uma oração é composta 
de elementos estruturais distintos no eixo sintagmático - um sujeito, um predicado, um objeto - mas no eixo paradigmático, pode-se escolher diferentes itens para se preencher estas categorias. $\mathrm{O}$ conceito de agnação diz respeito às relações associativas no eixo paradigmático.

\section{Considerações finais}

Apresentou-se, portanto, um breve panorama da interface entre a LSF e os estudos da tradução, bem como os pressupostos básicos daquela teoria. Importa ponderar que há alguns anos Baldry e Taylor (2001, p. 277) consideraram que "embora alguns artigos tenham sido publicados sobre o assunto, o interesse pelo papel da LSF nos estudos da tradução não chegou a se estabelecer como relevante dentro da área". ${ }^{12}$ Entretanto, Matthiessen (2007) retoma a questão para considerar que o intercâmbio recente entre pesquisadores de diferentes universidades, interessados em investigar a tradução sob a perspectiva da LSF, parece indicar o momento de uma fase mais produtiva nesta interface.

No Brasil, diversos estudiosos têm explorado eficazmente a interface entre a LSF e os estudos da tradução, produzindo pesquisas a partir de diferentes gêneros textuais, como por exemplo, Feitosa (2009) faz uma análise de legendas comerciais e legendas para fãs, utilizando os estudos sobre Fluxo da Informação; Araujo (2007) desenvolve um estudo descritivo do sistema de Projeção no português brasileiro, a partir do modelo da língua inglesa, utilizando um corpus de diferentes gêneros textuais; e Bueno (2005) analisa questões de criatividade lexical e Transitividade na tradução para o inglês do romance Macunaíma; entre outros.

A interface entre a LSF e os estudos da tradução, portanto, mostra-se produtiva para a análise do fenômeno tradutório, visto que a LSF é uma teoria abrangente que permite a análise e a descrição textual necessárias para a comparação de textos e línguas.

\footnotetext{
${ }^{12}$ “Interest in the role that systemic-functional linguistics might play in translation studies has never been feverish, though a number of articles have been written on the subject" (BALDRY;TAYLOR, 2001, p. 277, tradução nossa).
} 


\section{Referências}

ARAÚJO, C. G. O sistema semântico de PROJEÇÃo e sua dispersão gramatical em português brasileiro: uma descrição sistêmico-funcional orientada para os estudos linguísticos da tradução. Dissertação (Mestrado em Letras/Linguística Aplicada). Belo Horizonte: FALE/UFMG, 2007.

BALDRY, A., TAYLOR, C.. Computer assisted text analysis and translation: a functional approach in the analysis and translation of advertising texts. In: STEINER, E..; YALLOP, C.. (Ed.). Exploring translation and multilingual text production: beyond context. Berlim, New York: Mouton de Gruyter, 2001, p. 277-305.

BUENO, L. T. Transitividade, coesão e criatividade lexical em Macunaíma, de Andrade, e Macunaíma, de Goodland. Dissertação (Mestrado em Estudos Linguísticos). Belo Horizonte: Faculdade de Letras da UFMG, 2005.

CATFORD, J. C. A linguistic theory of translation. London: Oxford University Press, 1965. 103 p. (Language and Language Learning).

CATFORD, J. C. Uma teoria linguística da tradução: um ensaio de linguística aplicada. Trad. Centro de especialização de tradutores de inglês da PUC-Campinas. São Paulo: Cultrix: Pontificia Univ. Catolica de Campinas, 1980. 123p. (Tradução de: $A$ linguistic theory of translation).

EGGINS, S. An introduction to systemic functional linguistics. London \& New York: Continuum, 1994: 240-253.

FEITOSA, M. P.. Legendagem comercial e legendagem pirata: um estudo comparado. Tese (Doutorado em Letras/Linguística Aplicada). Belo Horizonte: FALE/UFMG, 2009.

FRANCIS, G., HUNSTON, S. Pattern grammar: a corpus-driven approach to the lexical grammar of English. Amsterdam/Philadelphia: John Benjamins, 2000.

GHADESSY, M. (Ed.). Text and context in functional linguistics. Amsterdam: John Benjamins, 1999. (Amsterdam studies in the theory and history of linguistic science. Series IV - Current issues in linguistic theory).

GHADESSY, M.. et al. (Ed.). Small corpus studies and ELT. Theory and practice. Amsterdam: John Benjamins, 2001. 
GHADESSY, M.., GAO, Y. Small corpora and translation. Comparing thematic organization in two languages. In: GHADESSY, Mohsen. et al. (Ed.). Small corpus studies and ELT. Theory and practice. Amsterdam: John Benjamins, 2001: 335-359.

GLEASON Jr, H. A. Lingustics and English grammar. New York: Hartford Seminary Foundation, 1965, $519 \mathrm{p}$.

GREGORY, M. What can linguistics learn from translation? In: STEINER, E., YALLOP, C. (Ed.). Exploring translation and multilingual text production: beyond content. Berlin, New York: Mouton de Gruyter, 2001:19-40.

HALLIDAY, M.A.K. et al. The linguistic sciences and language teaching. London: Longmans, 1964. 322 p. (Longmans' Linguistics Library).

HALLIDAY, M.A.K. et al. As ciências linguísticas e o ensino de línguas. Trad. Myriam Freire Morau. Petrópolis: Editora Vozes, 1974. 349 p. (Tradução de: The linguistic sciences and language teaching, 1964). Coleção Perspectivas Linguísticas, 12.

HALLIDAY, M.A.K. Corpus studies and probabilistic grammar.In: AIJMER, Karin., ALTENBERG, Bengt. (Ed.). English corpus linguistics: studies in honour of Jan Svartvik. London e New York: Longman, 1991, p. 30-43.

HALLIDAY, M.A.K. Language theory and translation practice. Rivista internazionale di tecnica del la traduzione. Università de Triesta, no. 1 (pilot issue), p. 15-25, 1992.

HALLIDAY, M.A.K. An introduction to functional grammar. 2. ed. London: Arnold, 1994.

HALLIDAY, M.A.K. Towards a theory of good translation. In: STEINER, Erich., YALLOP, Colin. (Ed.). Exploring translation and multilingual text production: beyond content. Berlin, New York: Mouton de Gruyter, 2001:13-18.

HALLIDAY, M.A.K., HASAN, R. Language, context, and text: aspects of language in a social-semiotic perspective. Geelong: Deakin Univesity, 1993, 126p.

HALLIDAY, M.A.K. e MATTHIESSEN, C. M. I. M. An introduction to functional grammar. 3rd edition, rev. ampl. London: Arnold, 2004, 689 p.

HOUSE, J. How do we know when a translation is good? In: STEINER, E., YALLOP, C. (Ed.). Exploring translation and multilingual text production: beyond content. Berlin \& New York: Mouton de Gruyter, 2001:127-160. 
HUNSTON, S. Corpora in applied linguistics. Cambridge: Cambridge University Press, 2002: 38-95.

JESUS, S. M. de. Representação do discurso e tradução: padrões de textualização em corpora paralelo e comparável. Dissertação (Mestrado em Letras/Linguística Aplicada). Belo Horizonte: Faculdade de Letras, UFMG/PosLin, 2004. 128 p.

JESUS, S M. de. Informática: o programa WordSmith Tools ${ }^{\circledR} 94$ In: GERBER, Regina; VASILEVSKI, Vera (Org.). Um percurso para pesquisas com base em corpus. Florianópolis: EDUFSC, 2007, p. 94-115. ISBN 978-85-328-0409-9

JESUS, S. M. de, PAGANO, Adriana Silvina. Probabilistic grammar in translation. In: BARBARA, Leila, BERBER SARDINHA, Tony (Ed.) Proceedings of the 33rd International Systemic Functional Congress (PUCSP, São Paulo, Brazil). Publicação

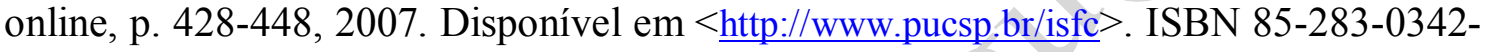
$\mathrm{X}$.

JESUS, S. M. de. Relações de tradução: SAY e DIZER em corpora de textos ficcionais. Belo Horizonte: Faculdade de Letras, UFMG/PosLin, 2008. Tese (Doutorado em Linguística Aplicada).

JOSEPH, J. E; LOVE, N.; TAYLOR, T. J. Landmarks in Linguistic thought II: the western tradition in the twentieth century. London: Routledge, 2001.

MARTIN, J. R. et al. Working with functional grammar. London: Arnold, 1997.

MATTHIESSEN, C. M.I.M. The environments of translation. In: STEINER, E., YALLOP, C. (Ed.). Exploring translation and multilingual text production: beyond content. Berlin, New York: Mouton de Gruyter, 2001, p. 41-124.

MATTHIESSEN, C. M.I.M. Multilingual humanity, multilingual studies: hope or despair. In: $32^{\text {th }}$ ISFC International systemic functional congress, 2005, Sidney. Resumoseletrônicos. Disponível em <http://www.asfla.org.au/isfc2005/speakers.html $>$. Acesso em $<21$ janeiro $2008>$.

MATTHIESSEN, C. M.I.M. Multilinguality: translation - a "feverish" phase in SFL? In: $34^{\text {th }}$ ISFC International systemic functional congress, 2007, Denmark. Resumoseletrônicos. Disponível em $<\underline{\mathrm{http}}$ ://www.humaniora.sdu.dk/isfc2007/matthiessen.htm $>$. Acesso em $<21$ janeiro $2008>$. 
NESBITT, C.; PLUM, G.. Probabilities in a systemic-functional grammar: the clause complex in English. In: FAWCETT, R., YOUNG, D. J. (Ed). New developments in systemic linguistics. London, New York: Printer Publishers, 1988. p. 6-38. (Theory and application, v. 2).

PAGANO, A., VASCONCELLOS, M. L.. Explorando interfaces: estudos da tradução, linguística sistêmico-funcional e linguística de corpus. In: ALVES, F., MAGALHÃES, C., e PAGANO, A. (Ed.). Competência em tradução: cognição e discurso. Belo Horizonte: Editora da UFMG, 2005, p.177-207. (Humanitas).

STEINER, E., YALLOP, C. (Ed.). Exploring translation and multilingual text production: beyond content. Berlin, New York: Mouton de Gruyter, 2001, 336p.

STEINER, E. Intralingual and interlingual versions of a text: how specific is the notion of translation. In: STEINER, E.; YALLOP, C. (Ed.). Exploring translation and multilingual text production: beyond context. Berlim, New York: Mouton de Gruyter, 2001, p. 161-190.

TEICH, E. Towards a model for the description of cross-linguistic divergence and commonality in translation. In: STEINER, E., YALLOP, C. (Ed.). Exploring translation and multilingual text production: beyond content. Berlin, New York: Mouton de Gruyter, 2001, p. 191-227.

TEICH, E. Cross-linguistic variation in system and text: a methodology for the investigation of translations and comparable texts. Berlin: Mouton de Gruyter, 2003, 276p. (Text, translation, computational processing, 5).

THOMPSON, G. Introducing functional grammar. London: Arnold, 1996.

VASCONCELLOS, M. L. Retextualizing "Dubliners": a systemic functional approach to translation quality assessment. Tese (Doutorado em Linguística Aplicada). Faculdade de Letras, UFSC, Florianópolis, 1997.

YALLOP, C. The construction of equivalence. In: STEINER, E., YALLOP, C. (Ed.). Exploring translation and multilingual text production: beyond content. Berlin, New York: Mouton de Gruyter, 2001, p. 229-246.

Artigo recebido em: 29.03.2012

Artigo aprovado em: 25.05.2012 